ARTICLE

Received 18 Feb 2016 | Accepted 26 Jul 2016 | Published 1 Sep 2016

DOl: $10.1038 /$ ncomms12707

OPEN

\title{
Structural basis underlying viral hijacking of a histone chaperone complex
}

Hongda Huang ${ }^{1, \star}$, Zhong Deng ${ }^{2, \star}$, Olga Vladimirova ${ }^{2}$, Andreas Wiedmer $^{2}$, Fang Lu ${ }^{2}$, Paul M. Lieberman ${ }^{2}$ $\&$ Dinshaw J. Patel ${ }^{1}$

The histone H3.3 chaperone DAXX is implicated in formation of heterochromatin and transcription silencing, especially for newly infecting DNA virus genomes entering the nucleus. Epstein-Barr virus (EBV) can efficiently establish stable latent infection as a chromatinized episome in the nucleus of infected cells. The EBV tegument BNRF1 is a DAXX-interacting protein required for the establishment of selective viral gene expression during latency. Here we report the structure of BNRF1 DAXX-interaction domain (DID) in complex with DAXX histone-binding domain (HBD) and histones H3.3-H4. BNRF1 DID contacts DAXX HBD and histones through non-conserved loops. The BNRF1-DAXX interface is responsible for BNRF1 localization to PML-nuclear bodies typically associated with host-antiviral resistance and transcriptional repression. Paradoxically, the interface is also required for selective transcription activation of viral latent cycle genes required for driving B-cell proliferation. These findings reveal molecular details of virus reprogramming of an antiviral histone chaperone to promote viral latency and cellular immortalization.

\footnotetext{
${ }^{1}$ Structural Biology Program, Memorial Sloan-Kettering Cancer Center, New York, New York 10065, USA. ${ }^{2}$ Gene Expression and Regulation Program, The Wistar Institute, Philadelphia, Pennsylvania 19104, USA. * These authors contributed equally to this work. Correspondence and requests for materials should be addressed to P.M.L. (email: lieberman@wistar.org) or to D.J.P. (email: pateld@mskcc.org).
} 
C hromatin assembly is a dynamic process that regulates all aspects of DNA biology, including transcription, replication and repair ${ }^{1}$. Histone chaperones perform a central function in the chromatin assembly process ${ }^{2-5}$. Several different classes of histone chaperones have been found to load specific histone variants to form distinct types of chromatin structures ${ }^{6,7}$. DAXX (death-domain associated protein-6) is a histone $\mathrm{H} 3.3$ chaperone that functions with the co-chaperone ATRX (ATP-dependent chromatin remodeller $\alpha$-thalassaemia $\mathrm{X}$-linked mental retardation protein) to assemble repressive heterochromatin at GC-rich repetitive elements, such as telomeres, pericentromeric regions and endogenous retrotransposons ${ }^{8-12}$. Chromatin organization can also function as a mechanism of resistance to viral infection ${ }^{13-15}$. Many viruses have acquired mechanisms to subvert the antiviral chromatin assembly factors, including DAXX and ATRX ${ }^{16}$. Importantly, each virus targets these factors in distinct ways that drive different infection strategies.

Epstein-Barr virus (EBV) is a human tumour virus that establishes long-term latent infection as a chromatinized episome ${ }^{14}$. During latent infection, EBV expresses a limited number of viral genes essential for viral genome persistence and host cell survival. The establishment of latency requires viral tegument proteins that are delivered with the viral $\mathrm{DNA}^{17}$. BNRF1 is an EBV-encoded tegument protein that is essential for transcription of viral genes during the earliest stages of viral infection ${ }^{18,19}$. BNRF1 is a member of a family of proteins with homology to the FGARAT enzyme involved in the de novo purine nucleotide biosynthesis ${ }^{14}$. Previously, we found that BNRF1 binds to DAXX to promote selective viral gene expression ${ }^{18,19}$. We now report the structural basis of how BNRF1 interacts with DAXX in a stable quaternary complex with histones $\mathrm{H} 3.3$ and $\mathrm{H} 4$. BNRF1 DAXX-interaction domain (DID) uses four extended loop domains not conserved in its cellular or viral orthologs, to contact all the components including DAXX histone-binding domain (HBD), H3.3 and $\mathrm{H} 4$ of the complex. We further show that the BNRF1-DAXX interface is responsible for BNRF1 localization to PML-nuclear bodies that are involved in host-antiviral resistance and transcriptional repression, and is also required for the selective transcription activation of viral latent cycle genes required for driving B-cell proliferation. These data reveal how the virus protein BNRF1 hijacks the cellular antiviral histone chaperone DAXX to promote viral latency and cellular immortalization.

\section{Results}

Structure of BRNF1 DID-DAXX HBD-H3.3-H4 complex. BNRF1 is a member of a family of $\gamma$-herpesvirus tegument proteins that share significant homology in their C-terminal domains to the cellular purine biosynthesis enzyme FGARAT (FGARAT) (Fig. 1a). The N-terminal domains of this family have acquired distinct capacities to bind various components of the host antiviral machinery, including SP100, DAXX and RIG-I (ref. 20). BNRF1 is unique among these viral proteins for its capacity to bind DAXX through an $\mathrm{N}$-terminal subdomain, termed the DID (aa 360-600) (Fig. 1a) ${ }^{18,19}$. On the basis of earlier findings by our groups and others revealing the structure of the ternary complex of the HBD of chaperone DAXX bound to the histone $\mathrm{H} 3.3-\mathrm{H} 4$ dimer $^{21,22}$, and biochemical evidence for quaternary complex formation between this ternary complex and the DID of BNRF1 (refs 18,19), we had successfully reconstituted and crystallized the quaternary complex of the BNRF1 DID (fragment 381-599; Fig. 1a) with the chaperone DAXX HBD and H3.3-H4 dimer. The structure of the quaternary complex shows that the BNRF1 DID folds into a globular $\alpha / \beta$ domain and is a
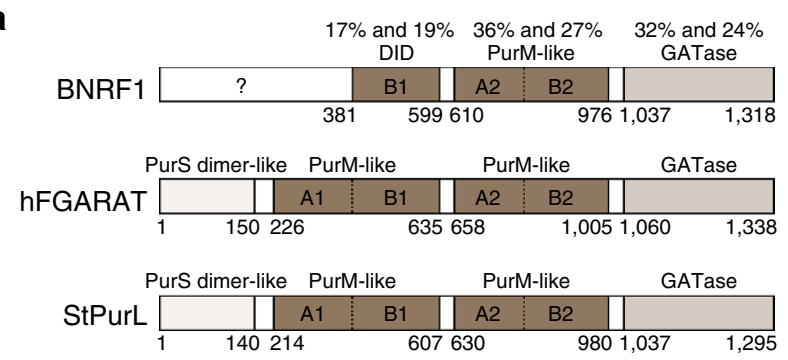

b
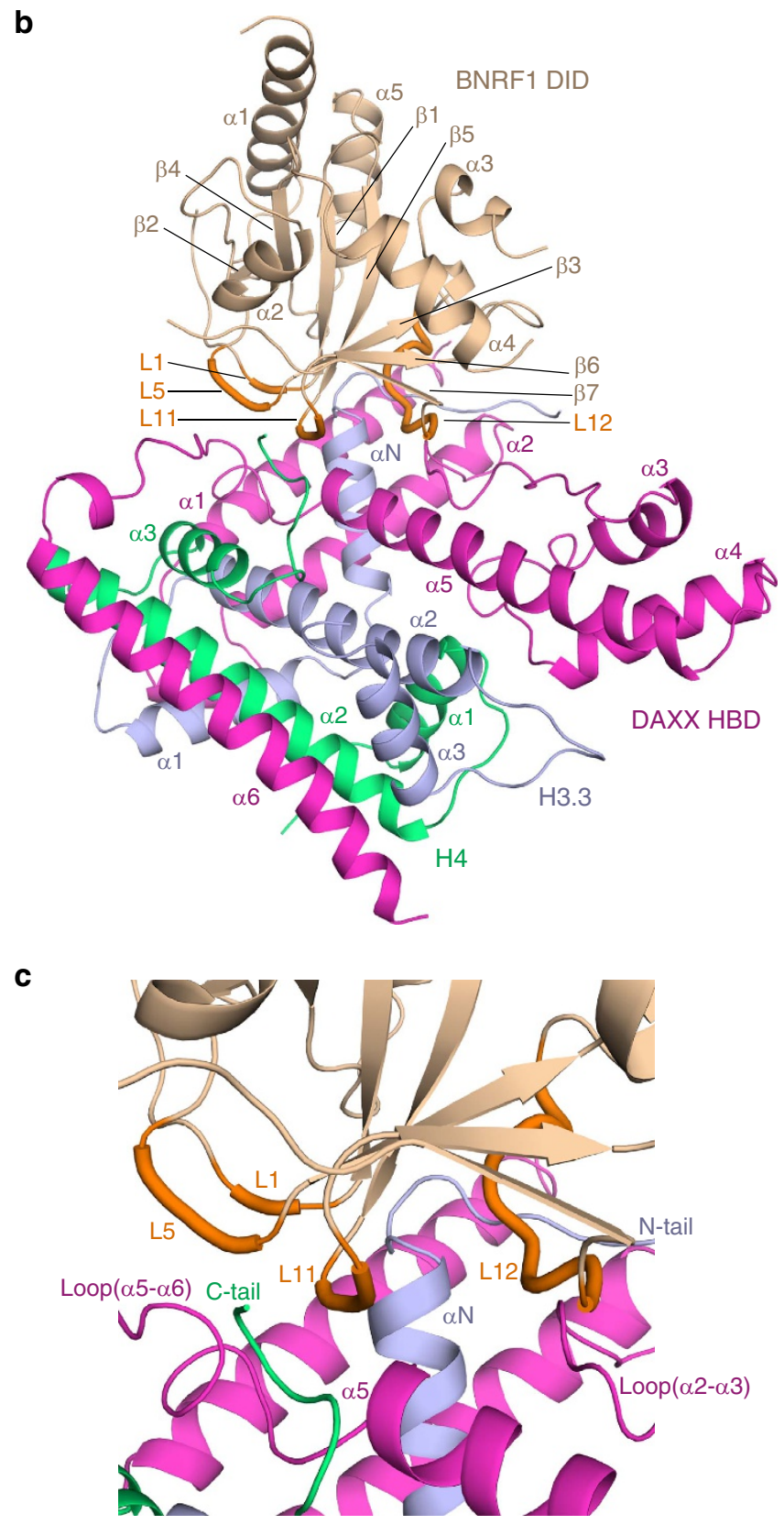

Figure 1 | Crystal structure of the quaternary BNRF1 DID-DAXX HBD-H3.3-H4 complex. (a) Domain structure of the BNRF1, human FGARAT (hFGARAT) and Salmonella typhimurium FGARAT (StPurL). The numbers show the sequence identities of BNRF1 with hFGARAT and StPurL, respectively. (b) Representative view of the overall structure of the quaternary BNRF1 DID-DAXX HBD-H3.3-H4 complex highlighting the L1, L5, L11 and L12 loops interacting with DAXX HBD-H3.3-H4 complex. (c) An expanded view of the intermolecular interface in the quaternary complex. 
positioned on top of the DAXX HBD-H3.3-H4 complex (Fig. 1b,c) with X-ray structural statistics listed in Table 1 and a portion of the electron density map shown in Supplementary Fig. 1a.

BNRF1 DID has seven anti-parallel $\beta$-strands ( $\beta 1$ to $\beta 7$ ) forming a $\beta$-sheet core fold, which is further surrounded on both sides by five $\alpha$-helices $(\alpha 1-\alpha 5)$ (Fig. 1b). A DALI search involving the fold of the BNRF1 DID alone based on the quaternary complex revealed that the overall fold is highly similar to the structure of the B-lobe of the first PurM-like domain of the Salmonella typhimurium FGARAT (StFGARAT or StPurL) protein ${ }^{23}$ (Fig. 1a), with an r.m.s.d. of $2.1 \AA$ (Supplementary Fig. 1b). It is also apparent that there are extensive local differences spread throughout the entire BNRF1 DID fold, especially in that the BNRF1 DID has an unique and extremely long loop (L12 loop connecting $\beta 6$ and $\beta 7$ ) (Supplementary Fig. 1b), which is important for binding to DAXX HBD-H3.3-H4 (see below). The DAXX HBD-H3.3-H4 portion of the structure of the quaternary BNRF1 DID-DAXX $\mathrm{HBD}-\mathrm{H} 3.3-\mathrm{H} 4$ complex, is similar to our previous structure of the ternary DAXX HBD-H3.3-H4 complex ${ }^{21}$, with small local differences in the binding interface for BNRF1 DID and with a small r.m.s.d. of $0.7 \AA$ (Fig. $1 \mathrm{~b}$ and Supplementary Fig. 1c).

Intermolecular contacts in the quaternary complex. The BNRF1 DID uses four loops (L1, L5, L11 and L12) to capture the chaperone DAXX HBD-H3.3-H4 complex through contacts to each component of this complex (Fig. 1b,c). Briefly, BNRF1 DID binds to DAXX HBD in the regions of the latter's C-terminal tip of the $\alpha 5$ helix, the loop connecting the $\alpha 5$ and $\alpha 6$ helices and the loop between the $\alpha 2$ and $\alpha 3$ helices. It also binds to histone $\mathrm{H} 3.3$ in the regions of the loop before the $\alpha \mathrm{N}$ helix and the base of the $\alpha \mathrm{N}$ helix (residues 40-52), and binds to histone $\mathrm{H} 4$ in its C-terminal tail (residues 99-102) (Fig. 1c).

The detailed interactions amongst BNRF1 DID and DAXX HBD-H3.3-H4 are shown in the Fig. 2a-c. A single-residue

\section{Table 1 | Data collection and refinement statistics.}

\begin{tabular}{|c|c|}
\hline & $\begin{array}{l}\text { BNRF1 DID-DAXX HBD-H3.3-H4 } \\
\text { complex }\end{array}$ \\
\hline \multicolumn{2}{|l|}{ Data collection } \\
\hline Space group & P32 21 \\
\hline \multicolumn{2}{|l|}{ Cell dimensions } \\
\hline$a, b, c(\AA)$ & $161.2,161.2,117.8$ \\
\hline$\alpha, \beta, \gamma\left(^{\circ}\right)$ & $90,90,120$ \\
\hline Resolution $(\AA)$ & $50-3.60(3.73-3.60)^{\star}$ \\
\hline$R_{\mathrm{pim}}(\%)$ & $7.3(93.8)$ \\
\hline$|/ \sigma|$ & $14.6(0.9)$ \\
\hline Completeness (\%) & $99.2(99.1)$ \\
\hline Redundancy & $11.0(10.7)$ \\
\hline \multicolumn{2}{|l|}{ Refinement } \\
\hline No. reflections (total/unique) & $226,960 / 20,715$ \\
\hline$R_{\text {work }} / R_{\text {free }}(\%)$ & $23.4 / 28.0$ \\
\hline \multicolumn{2}{|l|}{ No. atoms } \\
\hline Protein & 4,341 \\
\hline \multicolumn{2}{|l|}{ B-factors } \\
\hline Protein & 130.2 \\
\hline \multicolumn{2}{|l|}{ R.m.s deviations } \\
\hline Bond lengths $(\AA)$ & 0.013 \\
\hline Bond angles $\left(^{\circ}\right)$ & 1.68 \\
\hline
\end{tabular}

Tyr390 from L1 loop and three residues, Pro460, Lys461 and Gly462 from L5 loop of BNRF1 DID interact with the residues Asp342, Asp343, Tyr344 and Arg345 in the loop between $\alpha 5$ and $\alpha 6$ of DAXX HBD, with the Arg49 of H3.3 and with the H4 C-terminal tail (Fig. 2a). These intermolecular interactions include hydrogen bonds and extensive van der Waals contacts.

a

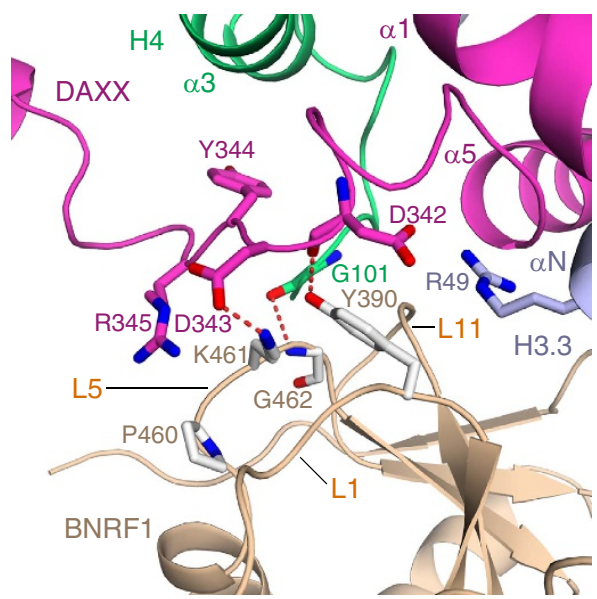

b

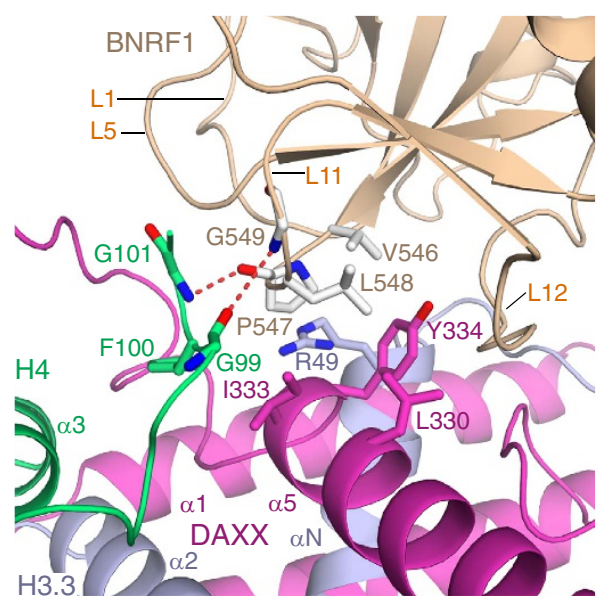

C

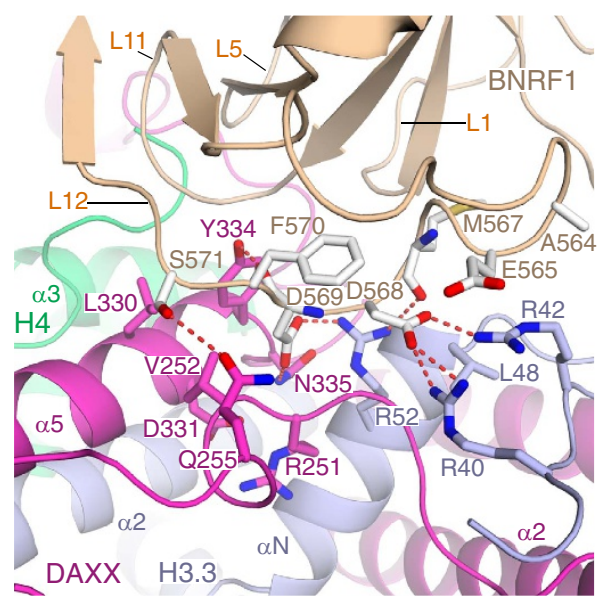

Figure 2 | Details of intermolecular interactions of the quartenary complex. (a-c) Each panel highlights the details of intermolecular interactions of the BNRF1 DID with the DAXX HBD-H3.3-H4 complex. The interactions of the L1 and L5 loops (a), the L11 loop (b) and of the L12 loop (c) of the BNRF1 DID with the DAXX HBD-H3.3-H4 complex. 
The residues Pro547 and Gly549 from the L11 loop of BNRF1 DID form hydrogen bonds with Gly101 and Gly99 of H4 tail, respectively (Fig. 2b). Moreover, the Leu548 of BNRF1 DID inserts into a hydrophobic pocket formed by residues Leu330, Ile333 and Tyr334 in the tip of the $\alpha 5$ helix of DAXX HBD (Fig. 2b).

The acidic residues Asp568 and Asp569 from L12 loop of BNRF1 DID form extensive hydrogen bonds and electrostatic interactions with the basic residues Arg40, Arg42 and Arg52 of H3.3, and form hydrogen bonds with Tyr334 and Asn335 of DAXX HBD (Fig. 2c). In addition, interactions of the L12 loop of BNRF1 DID with residues Arg251, Val252 and Gln255 in the loop between $\alpha 2$ and $\alpha 3$ helices of DAXX HBD may also contribute to binding.

Interfacial mutations influence the integrity of the complex. To validate the intermolecular interactions identified by the X-ray structure of the quaternary complex, we tested mutations in the BNRF1 interface for their effect on complex formation in vitro (Fig. 3a, b and Supplementary Fig. 2a, b), as well as in living cells (Fig. 3c-e and Supplementary Fig. 2c-e). We used in vitro pulldown assays to validate the observed intermolecular interactions between BNRF1 DID and the chaperone DAXX HBD-H3.3-H4 complex. The dual mutations V546D/L548D and D568A/D569A on BRNF1 DID (Fig. 3a), as well as R40A/R42A and R49A/R52A on $\mathrm{H} 3.3$ and Y334A on DAXX HBD (Fig. 3b) abrogate quaternary complex formation. Our data also show that dual mutations Y390A/K461A and V546S/L548S on BNRF1 DID, and D342A/D343A on DAXX HBD partially diminish, but do not eliminate complex formation in vitro.

We next assayed by immunoprecipitation (IP) the ability of FLAG-tagged BNRF1 or site-directed mutants for interaction with DAXX in IPs with either FLAG or DAXX antibody after transfection into $293 \mathrm{HEK}$ cells. Substitution mutations in BNRF1 containing either Y390A, V546A/L548A, V546S/L548S, D568A/D569A or Y390A/K461A severely disrupted BNRF1DAXX interaction, with the D568A/D569A mutant causing instability in BNRF1 (Fig. 3c and Supplementary Fig. 2c). Mutant K461A had a modest effect on BNRF1-DAXX interaction. Similar results were observed when HA-DAXX and FLAG-BNRF1 wt or mutants were co-transfected into 293HEK cells and assayed in IPs with either FLAG or HA antibody (Supplementary Fig. 2d). Mutations in the DAXX interface at positions D342A/D343A or $\mathrm{Y} 334 \mathrm{~A}$ disrupted the interaction with BNRF1 in both FLAG-BNRF1 and HA-DAXX IPs (Fig. 3d). The contribution of histone $\mathrm{H} 3.3$ was assayed by co-transfection of FLAG-H3.3, GFP-BNRF1 and HA-DAXX into 293HEK cells. Mutations in histone $\mathrm{H} 3.3$ at positions $\mathrm{R} 40 \mathrm{~A} / \mathrm{R} 42 \mathrm{~A}$ or R49A/R52A disrupted the interaction with GFP-BNRF1 in FLAG-H3.3 IP experiments (Fig. 3e). These mutations reduced, but did not eliminate their interaction with HA-DAXX either in the presence or absence of GFP-BNRF1, with R40A/R42A causing instability in histone H3.3 (Fig. $3 \mathrm{e}$ and Supplementary Fig. 2e). These findings with full-length BRNF1 and DAXX in living cells substantiate the importance of the interfacial contacts determined by the structure for maintaining a quaternary complex in living cells.

Mutants on BNRF1 reduce its co-localization with DAXX. We next assayed the importance of the BNRF1-DAXX interface on BNRF1 subcellular localization (Fig. 4a-c). BNRF1 wt co-localized strongly with DAXX at punctate subnuclear structures previously shown to be PML-NBs (promyelocytic leukemia-nuclear bodies). BNRF1 mutations at K461A, Y390A/ K461A and V546S/L548S reduced, but did not eliminate BNRF1 co-localization at PML-NBs (Fig. 4a). Mutations at
Y390A, V546A/L548A and D568A/569A showed a diffuse cytoplasmic pattern and severely reduced the frequency of BNRF1 co-localization with DAXX at PML-NBs (Fig. 4b). Biochemical fractionation studies, which do not involve the wash conditions used for microscopy, indicated that BNRF1 and DAXX have both cytoplasmic and nuclear distributions, and that mutations in the DAXX interface did not alter this overall distribution (Supplementary Fig. 3). These findings indicate that BNRF1 interfacial mutants were not compromised for nuclear localization, but BNRF1 localization to PML-NBs depends on its interaction with DAXX.

Functional significance of BNRF1-DAXX interaction. We next tested the functional significance of the BNRF-DAXX interaction by generating recombinant EBV genomes with either D568A/D569A (Figs 5 and 6) or V546D/L548D (Fig. 6 and Supplementary Fig. 4), or by trans-complementation of a BNRF1 deletion virus with either BNRF1 wt, Y390A, V546A/L548A or Y390A/K461A (Fig. 6). Viruses generated from these different sources were validated for integrity by restriction digest and Southern blot for terminal and internal repeat sequences (Fig. 5a and Supplementary Fig. 4a), as well as for viral gene expression by IF and western blot (WB) (Fig. 5b, c and Supplementary Figs 4b,c and $5 \mathrm{a}$ ). Virus produced from these cells was normalized for viral DNA copies by quantitative PCR and formation of GFP-positive Raji cells after infection (Fig. 5d and Supplementary Fig. 4d). Virion proteins were further examined by WB, and we found that both wt and BNRF1 mutant viruses contain comparable level of viral BNRF1, BALF2 and cellular protein Actin, all of which have previously been identified in proteomic studies of EBV virions ${ }^{24}$ (Figs 5e and $6 \mathrm{~g}$ and Supplementary Fig. 4e). We did not detect any western signals for these proteins in mock preparations from HEK-293T cells, indicating that the virus preparations are specific for the virus production cells.

To determine the functional significance of BNRF1-DAXX interaction, quantitative PCR normalized units of wt and BNFR1 mutant viruses were used to infect primary human B-lymphocytes (Fig. 6). Viral gene expression was assayed by quantitative real-time PCR at 1 week post infection. We found that all BNRF1 mutations that compromised DAXX binding were defective for latency-associated transcription (for example, EBNA1, EBNA2, EBNA3C mRNA) and cellular proliferation marker Ki67 relative to wt BNRF1 virus (Fig. 6a,b,f and Supplementary Fig. 5b). Interestingly, recombinant viruscontaining D568A/569A and V546D/L548D mutations showed elevated levels of ZTA immediate early gene transcripts, suggesting that BNRF1-DAXX interaction is also required for suppression of early lytic cycle gene activity. The similar pattern of changes in viral gene expression was observed at $72 \mathrm{~h}$ after viral infection (Supplementary Fig. 5c). Moreover, neither D568A/L569A nor V546D/L548D were capable of inducing lymphoblast proliferation and colony formation similar to wt EBV, indicating that the BNRF1-DAXX interaction is essential for B-cell immortalization (Fig. 6c-e).

\section{Discussion}

Our studies indicate that BRNF1 DID contacts all three components of the chaperone complex, namely DAXX HBD, H3.3 and H4 (Fig. 1b). A network of intermolecular hydrogen bonds, electrostatic and hydrophobic interactions mediate recognition at the interface in the quaternary complex (Fig. 2). The in vitro and cell-based mutational studies (Fig. 3) emphasize the importance of the interactions of the acidic L12 loop (Asp568 and Asp569) of BNRF1 DID with the basic regions (Arg40, Arg42, Arg49 and Arg52) of H3.3, given loss in binding 
a

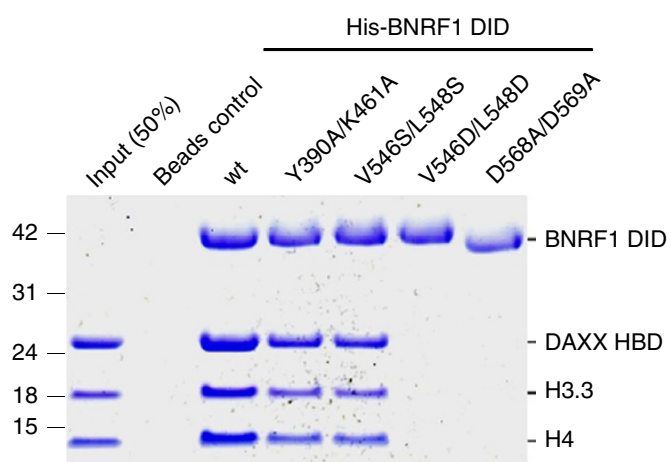

C

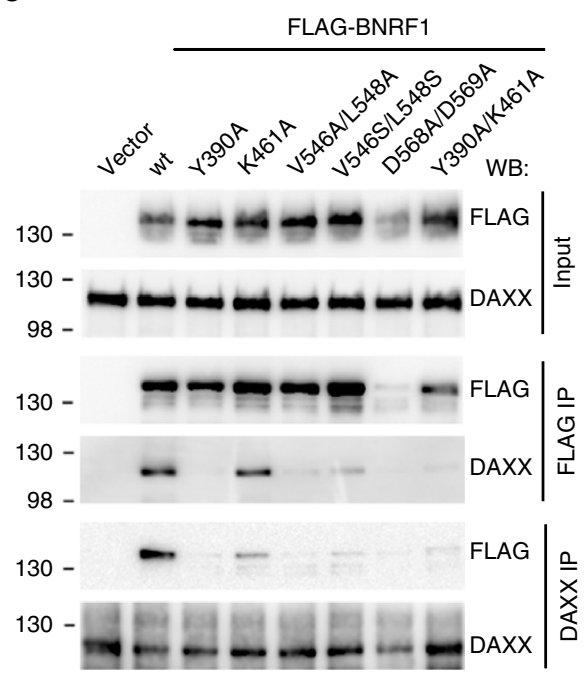

b

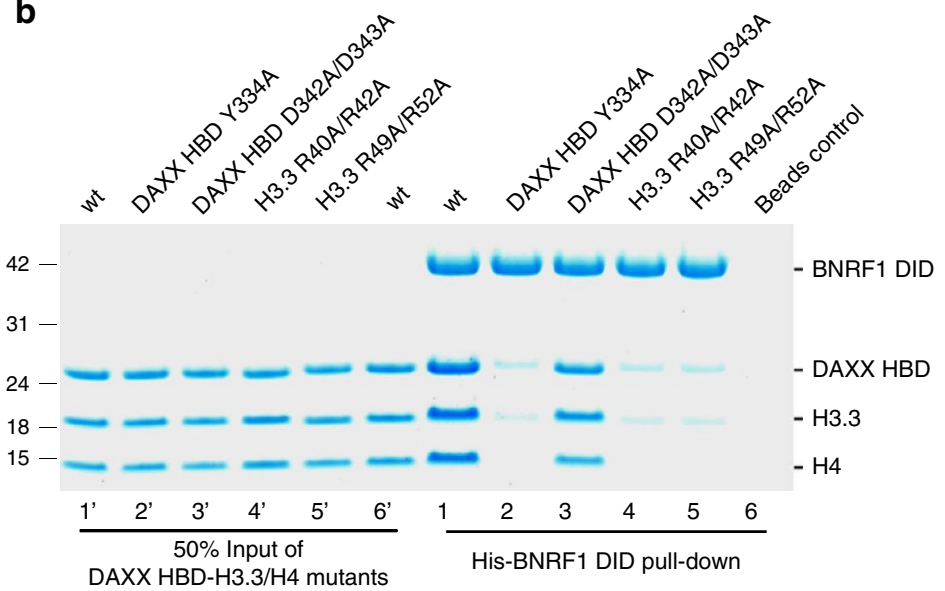

d

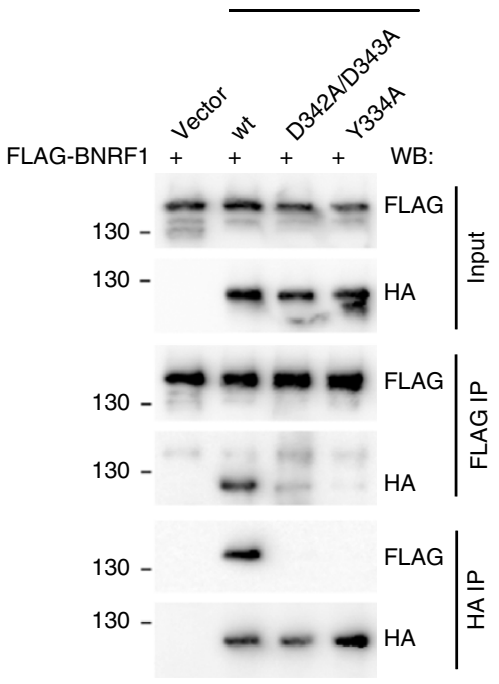

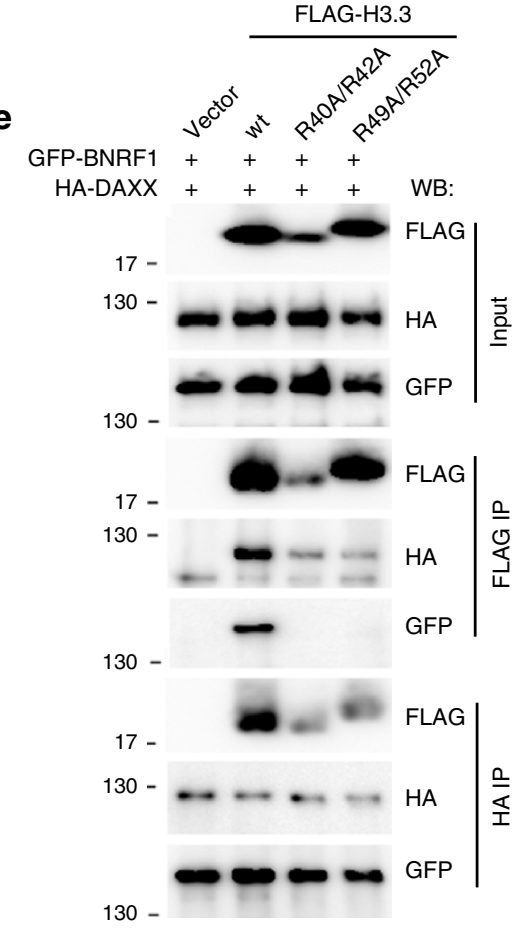

Figure 3 | Impact of interfacial mutants on the BNRF1-DAXX-H3.3-H4 complex in vitro and in living cells. (a) In vitro pulldown of the BNRF1 DID wild-type (wt) and mutants with the DAXX HBD-H3.3-H4 complex. (b) In vitro pulldown of the BNRF1 DID wt with mutants of the DAXX-H3.3-H4 complex. The purifications of the mutants of the DAXX HBD-H3.3-H4 complex are shown in the Supplementary Fig. 2a,b. (c) IP-Western analysis of HEK293 cells transfected with expression plasmids for FLAG-BNRF1 wt or mutants, as indicated above each lane. Protein complexes were isolated by FLAG- or DAXX-IP and assayed by WB with either FLAG or DAXX antibody, as indicated. Input (10\%) for FLAG and DAXX is shown in top panels. Molecular weight markers are indicated in $\mathrm{kDa}$. (d) Same as in c, but HA-DAXX, or HA-DAXX mutants (as indicated) were co-transfected with FLAG-BNRF1 wt and subject to IP with either FLAG beads or HA antibody followed by WB with HA or FLAG antibodies, as indicated. (e) Same as in c, but FLAG-H3.3 or H3.3 mutants (as indicated) were co-transfected with GFP-BNRF1 and HA-DAXX, and then subject to IP with either FLAG beads or HA antibody, and assayed by WB with FLAG, HA or GFP antibody, as indicated. Gel source data, please see Supplementary Fig. 7.

on Ala substitution at these positions. Further, studies with the Y334A mutant emphasize that Tyr334 of DAXX HBD is also important for the binding with BNRF1 DID. The above intermolecular recognition elements in our structure of the quaternary BNRF1 DID-DAXX HBD-H3.3-H4 complex and their response to interfacial mutations provide definitive insights into the structural basis underlying how BNRF1 interacts with the histone chaperone DAXX-H3.3-H4 complex.

Our work also reveals that BNRF1 interfacial mutants were not compromised for nuclear localization, but BNRF1 localization to PML-NBs depends on its interaction with DAXX (Fig. 4). Recombinant viruses harbouring either D568A/D569A or
V546D/L548D mutations show defects in activating viral latent gene expression and driving $\mathrm{B}$ cell proliferation, suggesting that BNRF1 localization to PML-NBs mediated by BNRF1-DAXX interface is required for the establishment of viral latency program during primary infection (Fig. 6). Our analysis of these bacmids by restriction digest and Southern blot for terminal and internal repeat sequences showed that BNRF1 mutations did not alter the integrity of viral genome (Fig. 5). In addition, WB of viral reactivation from virus production cells revealed that recombinant viruses harbouring the BNRF1 mutations were induced at a comparable level of viral gene expression as the wild-type viruses. However, WB analysis of virion proteins 
a

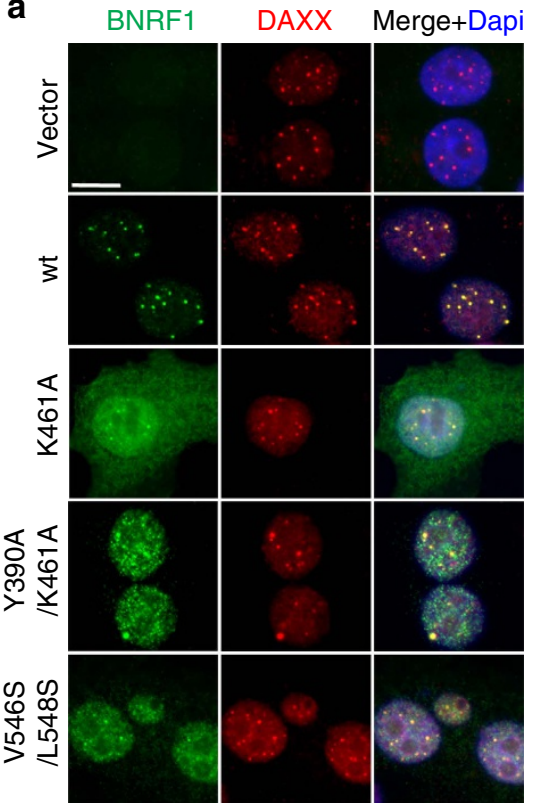

b

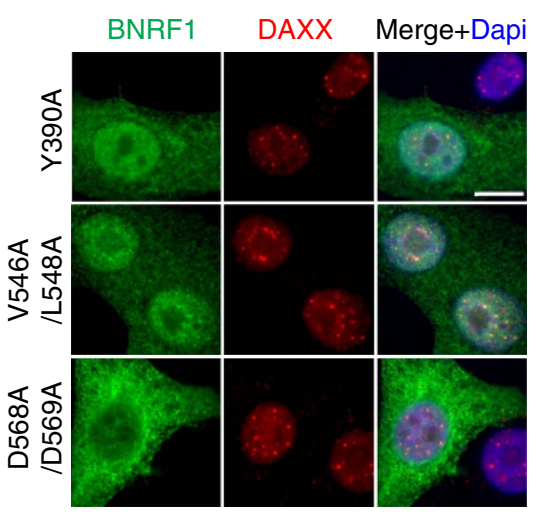

C

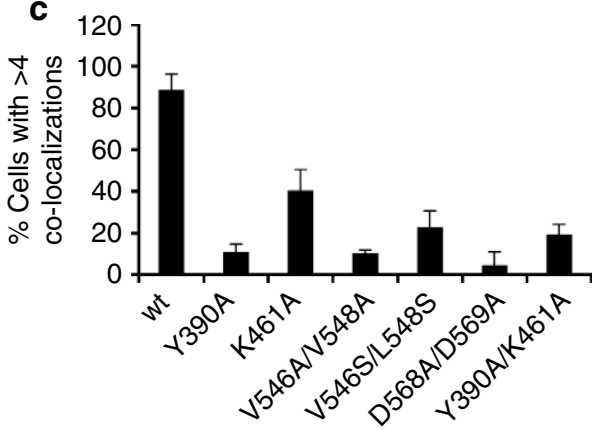

Figure 4 | Interfacial mutants on BNRF1 exhibit reduced subnuclear co-localization with DAXX. (a,b) HEP2 cells were transfected with FLAG-BNRF1 wt or mutants (as indicated) for $24 \mathrm{~h}$ and then assayed by IF with antibodies to FLAG (green), DAXX (red) and Dapi (blue) in Merge image. Scale bar, $20 \mu \mathrm{m}$. (c) Quantification of cells with BNRF1 foci co-localizing with DAXX foci. Cells were scored positive only if $>4$ BNRF1 foci co-localized with DAXX foci. The bar graph shows means \pm s.d. derived from quantification of over 100 nuclei for each transfection from multiple independent IF assays $(n=4)$.

indicated that BNRF1 mutant viruses D568A/D569A and V546D/L548D expressed increased levels of BALF2 and BNRF1, respectively relative to their wild-type viruses. While increased levels of BALF2 or BNRF1 in mutant virions are unlikely to lead to the reduction in viral latent gene expression during primary infection, we cannot exclude the possibility that BNRF1 mutations alter the composition and assembly of virions, which in turn affect infectivity, viral gene expression, and cellular proliferation. Future experiments including electron microscopy of virions will be necessary for validating that BNRF1 mutations modulate viral gene expression by lack of interaction with DAXX, but not by the structural changes in mutant virions.

While our data clearly demonstrated that mutations in BNRF1/DAXX interface disrupt BNRF1 and DAXX interactions both in vitro and in vivo, the underlying mechanisms how BNRF1 mutations compromise viral infection into primary $B$ cells remains unclear. Our earlier studies suggested that BNRF1 activates early gene transcription through the disruption of ATRX-DAXX interaction and the establishment of a chromatin structure permissive for viral early gene activity ${ }^{18,19}$. To this end, future studies by ChIP analysis of ATRX/DAXX occupancy and chromatin assembly at viral genomes after primary infection with BNRF1 mutant or wild-type viruses will be necessary to elucidate how BNRF1-DAXX interactions contribute to the establishment of viral latency.

Notably, despite EBV and KSHV being in the same family of $\gamma$-herpesvirus, the BNRF1 of EBV interacts with the DAXX-H3.3H4 complex, while its KSHV homologue ORF75 does not ${ }^{20}$. The sequence alignment of these two species reveals that the BNRF1 residues identified by our structure to be important for binding to the DAXX-H3.3-H4 complex are not conserved in KSHV ORF75 (Supplementary Fig. 6), thus explaining why BRNF1 but not KSHV ORF75 interacts with the DAXX-
H3.3-H4 complex. Importantly, the BNRF1 interaction with DAXX-H3.3-H4 is essential for BNRF1 localization to PML-NBs and the selective activation of EBV latent, but not lytic cycle gene transcription. These data support the model that BNRF1 reprograms DAXX histone chaperone function to promote the exclusive expression of EBV latent cycle genes essential for EBV induced B-cell proliferation and immortalization.

\section{Methods}

Protein expression and purification. The cDNA of human DAXX HBD (178-389) was cloned into a modified RSFDuet-1 vector (Novagen), while the cDNA of human histones $\mathrm{H} 3.3$ and $\mathrm{H} 4$ were cloned into one pETDuet vector (Novagen). The DAXX HBD (178-389)-H3.3-H4 ternary complex was coexpressed and prepared in Escherichia coli ${ }^{21}$. The cDNA of EBV BNRF1 DID (381-599) was synthesized at GENEWIZ and was cloned into a modified RSFDuet-1 vector (Novagen), with an N-terminal His ${ }_{6}$-SUMO tag. The BNRF1 DID was expressed in BL21(DE3)-RIL cell strain (Stratagene). The expressed protein complex was first purified on nickel-sepharose affinity column (GE Healthcare). After removing the $\mathrm{His}_{6}$-SUMO tag by using Ulp1 (SUMO protease), the protein complex was further purified on HiLoad 16/600 Superdex 200 column (GE Healthcare). Equal moles of the BNRF1 DID protein and the DAXX HBD-H3.3-H4 ternary complex were mixed together and incubated on ice for $10 \mathrm{~min}$ (mins) to form the quaternary BNRF1 DID-DAXX HBD-H3.3-H4 complex, then the quaternary complex was purified with HiLoad 16/600 Superdex 200 column. The resulting quaternary complex was concentrated to $5.8 \mathrm{mg} \mathrm{ml}^{-1}$ in a buffer of $20 \mathrm{mM}$ Tris $\mathrm{pH} 7.5$ and $1 \mathrm{M} \mathrm{NaCl}$.

Crystallization. The quaternary BNRF1 DID-DAXX HBD-H3.3-H4 complex in a concentration of $5.8 \mathrm{mg} \mathrm{ml}^{-1}$ was crystallized in $0.1 \mathrm{M} \mathrm{MES} \mathrm{pH} 6.0,0.8 \mathrm{M}$ ammonium sulfate using sitting-drop vapour-diffusion method at $20^{\circ} \mathrm{C}$. All the crystals were soaked in a cryoprotectant made from mother liquor supplemented with $20 \%$ glycerol before flash freezing in liquid nitrogen.

Structure determination. The data sets for the quaternary BNRF1 DID-DAXX HBD-H3.3-H4 complex were collected at $0.979 \AA$ on $24-\mathrm{ID}-\mathrm{C} / \mathrm{E}$ NE-CAT (Advanced Photo Source, Argonne National Laboratory). All the data sets were processed by using the HKL 2,000 program. The initial structure for the quaternary 
a

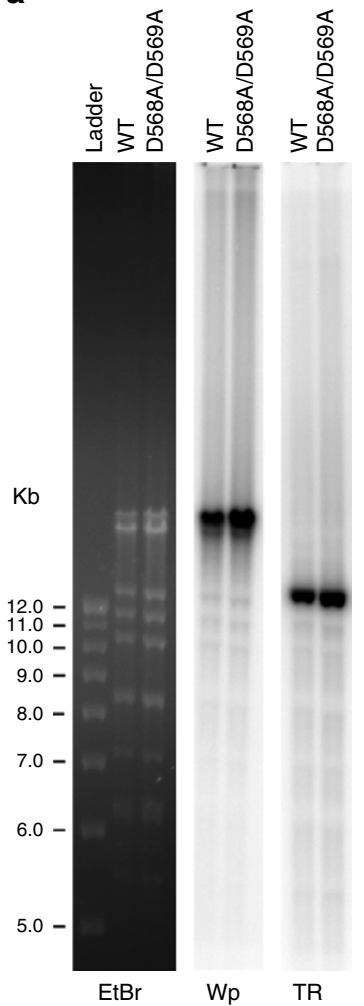

b
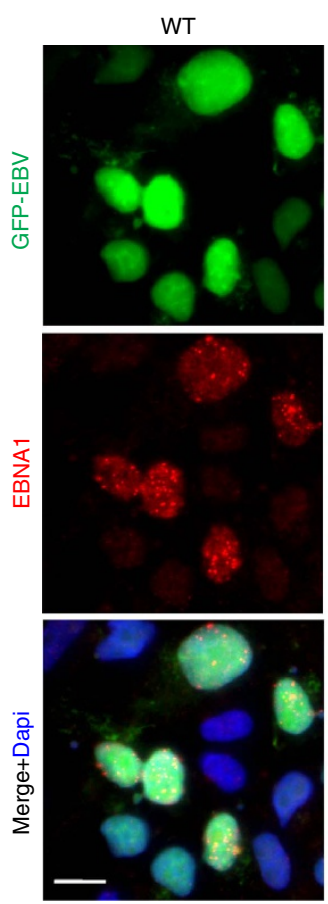

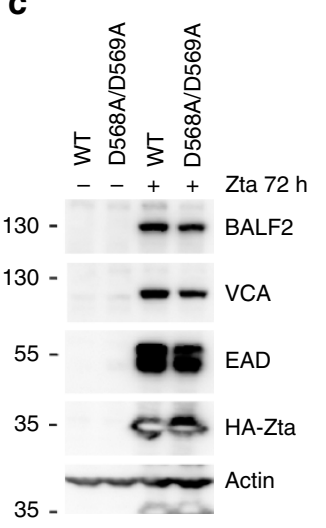

e

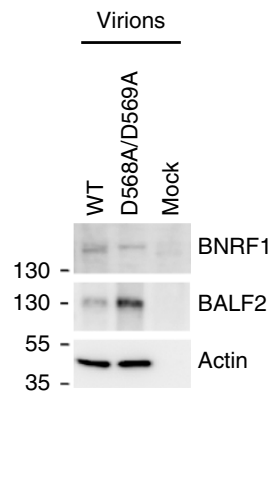

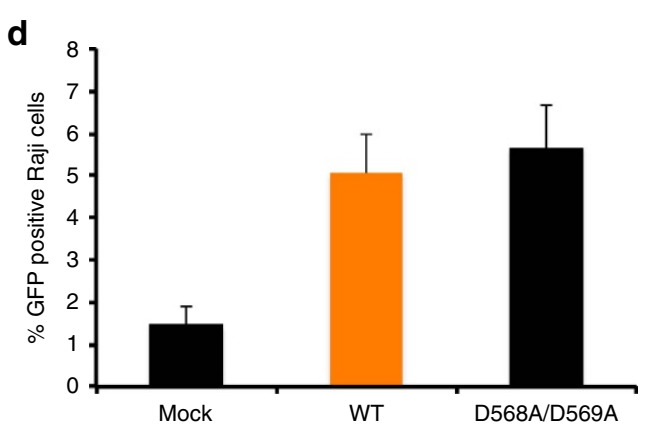

Figure 5 | Generation of the recombinant EBV-GFP BNRF1 D568A/D569A bacmid and virus. (a) BACEBV-GFPwt and BNRF1D568A/D569A mutant was digested with EcoRl, fractionated on $0.8 \%$ agarose gel, and stained with ethidium bromide (EtBr) followed by Southern blots with ${ }^{32} \mathrm{P}$-labelled probes specific for Wp or TR regions. (b) Immunofluorescence analysis for EBNA1 (red) in BACEBV-GFPwt and BNRF1D568A/D569A HEK-293T virus production cell lines. GFP expression levels (green) were monitored and Dapi (blue) was shown in Merge image. Scale bar, $10 \mu \mathrm{m}$. (c) Virus production cells shown in $\mathbf{b}$ were either mock transfected or co-transfected with expression vectors for HA-tagged Zta and BALF4 for $72 \mathrm{~h}$. Viral reactivation was examined by WB with BALF2, VCA, EAD, HA or actin antibody, as indicated. (d) Raji cells were mock treated or superinfected with recombinant EBV wt (orange) or BNRF1 D568A/D569A genomes at a MOI of 30 for 4 days in the presence of $100 \mathrm{ng} \mathrm{ml}^{-1}$ TPA and then assayed for Raji cell infection by FACS analysis of GFP-positive cells. The bar graph represents means \pm s.d. $(n=3)$. (e) Virions from HEK-293T mock cells or recombinant wt or D568A/D569A virus production cells were assayed by WB with antibodies to BNRF1, BALF2 or actin. FACS, fluorescence-activated cell sorting; MOI, multiplicity of infection; TPA, tetradecanoyl phorbol acetate.

BNRF1 DID-DAXX HBD-H3.3-H4 complex was solved by molecular replacement in PHASER ${ }^{25}$ with the structure of the ternary DAXX HBD-H3.3-H4 complex (PDB 4H9N) as a search model and manually refined and built using $\mathrm{Coot}^{26}$. The final structure of this complex was refined to $3.60 \AA$ resolution using PHENIX ${ }^{27}$. The Ramachandran plot was calculated with PHENIX and showed 93.8\% favoured and $6.2 \%$ allowed. Table 1 summarizes the statistics for data collection and structural refinement.

Cells for this study. Hep2, 293HEK, and 293T cells were obtained from ATCC and grown in Dulbecco's modified Eagle medium (DMEM) supplemented with $10 \%$ fetal bovine serum (FBS), $100 \mathrm{U} \mathrm{ml}^{-1}$ penicillin-streptomycin. All these cell lines were validated to be free of mycoplasma contamination and were used for transfection experiments. Raji cells were validated for EBV genome integrity and grown in RPMI 1640 medium supplemented with $10 \% \mathrm{FBS}, 100 \mathrm{U} \mathrm{ml}^{-1}$ penicillin-streptomycin. Purified human primary B cells were purchased from Path BioResource (Department of Pathology and Laboratory Medicine, University of Pennsylvania). Primary B cells isolated from various blood donors, including ND388, ND365, ND473, ND317, ND343 and ND422, were cultured in RPMI 1640 medium supplemented with $20 \%$ FBS, $10 \mathrm{mM}$ Hepes, $\mathrm{pH} 7.2,1 \mathrm{mM}$ Sodium Pyruvate, $10 \mathrm{mM}$ GlutaMAX (Gibco) and $100 \mathrm{U} \mathrm{ml}^{-1}$ penicillin-streptomycin. All cells were grown in a $5 \% \mathrm{CO}_{2}$ incubator at $37^{\circ} \mathrm{C}$.

Cloning and mutagenesis. pcDNA3-HA-DAXX was a generous gift from H.-M. $\mathrm{Shih}^{28}$, and FLAG-BNRF1 expression vectors were generated by PCR amplification and cloning into HindIII/SalI sites of the p3xFLAG-Myc-CMV-24 Expression Vector (Sigma-Aldrich) ${ }^{18}$. An EGFP-BNRF1 expression plasmid was generated by PCR amplification and cloning into HindIII/SalI sites of pEGFP-C3 vector (Clonetech). A FLAG-H3.3 expression vector was constructed by PCR amplification and cloning into HindIII/BamHI sites of p3xFLAG-CMV vector
(Sigma). FLAG-BNRF1 mutants (Y390A, K461A, V546A/L548A, V546S/L548S, D568A/D569A and Y390A/K461A), HA-DAXX mutants (D342A/D343A and Y334A), and FLAG-H3.3 mutants (R40A/R42A and R49A/R52A) were generated by QuickChange Site-directed Mutagenesis (Stratagene). All constructs were verified by sequencing and cloning details are available upon request.

Co-Immunoprecipitation. Co-Immunoprecipitation (Co-IP) was performed in HEK293 cells transfected with indicated expression plasmids using Lipofectamine 2000 (Invitrogen). Cells were harvested $48 \mathrm{~h}$ post transfection, washed two times with cold PBS, and then lysed in cold lysis buffer $(20 \mathrm{mM}$ Tris, pH 8.0, $137 \mathrm{mM}$ $\mathrm{KCl}, 1 \mathrm{mM}$ EDTA, $1.5 \mathrm{mM} \mathrm{MgCl} 2,10 \%$ Glycerol, and $1 \%$ Triton X-100 supplemented with $1 \mathrm{mM}$ DTT and $0.1 \%$ mammalian protease inhibitor cocktail mix) for $30 \mathrm{~min}$ on ice. Cell lysates were centrifuged at about $18,000 \mathrm{~g}$ for $10 \mathrm{~min}$, and the supernatants were precleared with Protein G Sepharose beads (GE Healthcare) for $60 \mathrm{~min}$ at $4^{\circ} \mathrm{C}$ with rotation. One $\mathrm{ml}$ of precleared lysates $\left(\sim 5 \times 10^{6}\right.$ cells $)$ were immunoprecipitated with either FLAG agarose beads (Sigma) or mouse monoclonal anti-HA (12CA5, Roche) or rabbit anti-DAXX (Sigma) overnight at $4^{\circ} \mathrm{C}$ with rotation. For HA and DAXX IP, the immuno-complex was collected with Protein G sepharose beads with rotating at $4{ }^{\circ} \mathrm{C}$ for $3 \mathrm{~h}$. The beads were washed three times with BC300 or BC500 (500 mM KCl, $20 \mathrm{mM}$ Tris- $\mathrm{HCl}, \mathrm{pH} 8.0,0.2 \mathrm{mM}$ EDTA, $10 \%$ glycerol and $10 \mathrm{mM} \beta$-mercaptoethanol) for endogenous DAXX or for HA-DAXX, respectively, and then followed by once with BC100 at $4{ }^{\circ} \mathrm{C}$. Pulled down proteins were eluted by either FLAG peptide or boiling with $2 \times$ Laemmli buffer (100 mM Tris-HCl, pH 6.8, 4\% SDS, 0.2\% Bromophenol Blue, and 20\% Glycerol), and were subject to SDS-polyacrylamide gel electrophoresis (8-16\% Tris-Glycine gel, Invitrogen) and WB analysis.

Indirect immunofluorescence. Indirect immunofluorescence (IF) was performed in Hep2 cells transfected with BNRF1 expression plasmids using Lipofectamine 
2000 (Invitrogen). Transfected cells were reseeded in 24-well plates with coverslips at $6 \mathrm{~h}$ post transfection. At $24 \mathrm{~h}$ post transfection, cells were rinsed in $1 \times \mathrm{PBS}$, fixed with $1 \%$ paraformaldehyde in $1 \times \mathrm{PBS}$ at room temperature for $15 \mathrm{~min}$, and permeablized with $0.3 \%$ Triton-X 100 for another $15 \mathrm{~min}$. Coverslips were blocked in PBG solution $(0.2 \%$ cold water fish gelatin, $0.5 \%$ BSA in $1 \times$ PBS $)$ for $30 \mathrm{~min}$ at room temperature. Cells were then stained sequentially with mouse monoclonal anti-FLAG (F1804, Sigma, 1:10,000 dilution in PBG) followed by rabbit anti-DAXX
(F7810, Sigma, 1:800 dilution in PBG) at room temperature for $1 \mathrm{~h}$ each. Secondary antibody stainings were performed at toom temperature for $1 \mathrm{~h}$ with Alexafluor488 goat anti-mouse antibody and Alexafluor594 goat anti-rabbit antibody (Invitrogen) at 1:800 dilution in PBG, respectively. Nuclei were counterstained with

$0.1 \mathrm{mg} \mathrm{ml}^{-1}$ DAPI in PBG and slides were mounted with FluoroMount-G

(SouthernBiotech). Images were captured with a $100 \times$ objective lens on a Nikon E600 Upright microscope (Nikon Instruments, Inc., Melville, NY, USA) using a
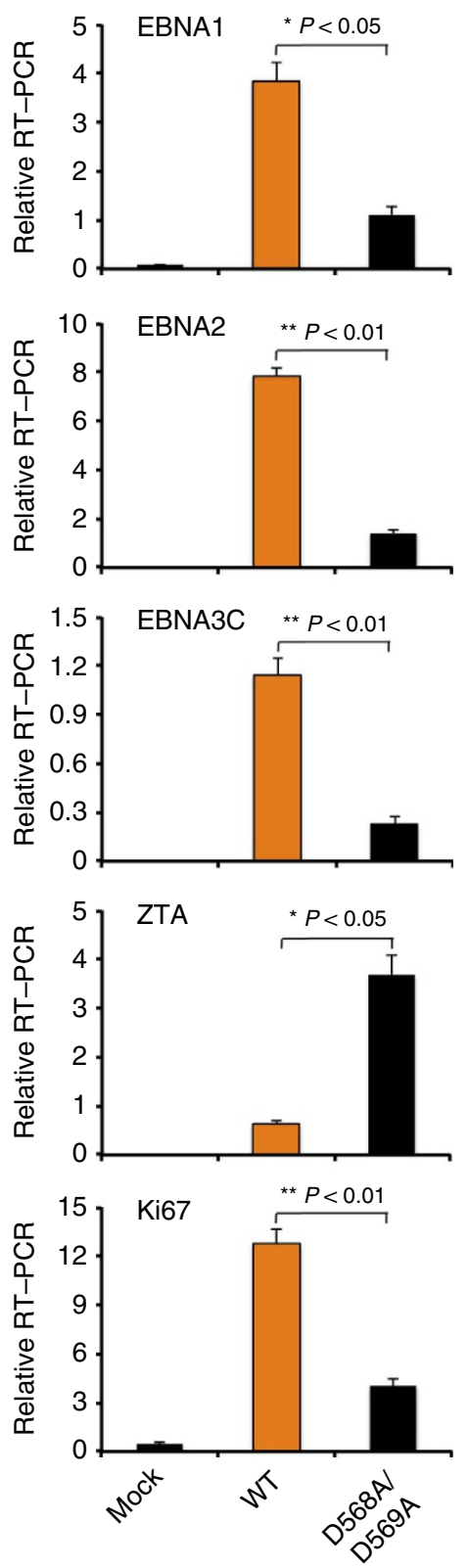

f

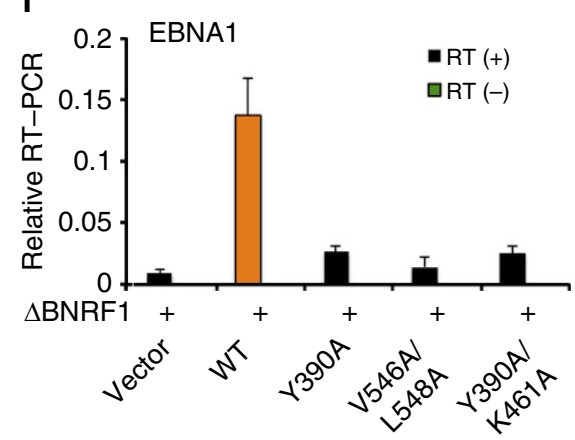

b
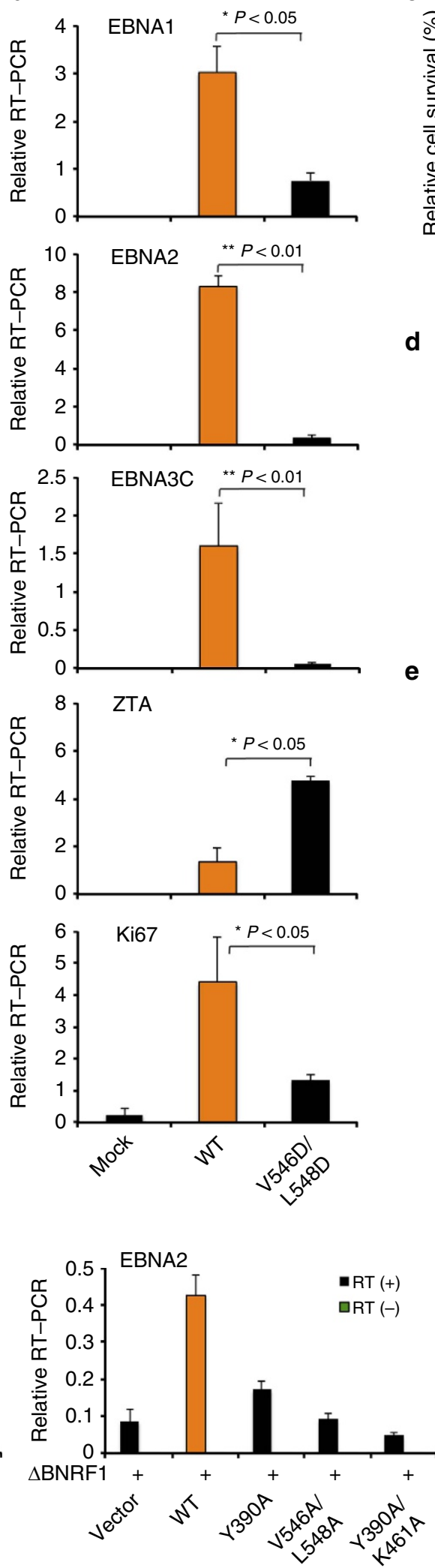

C

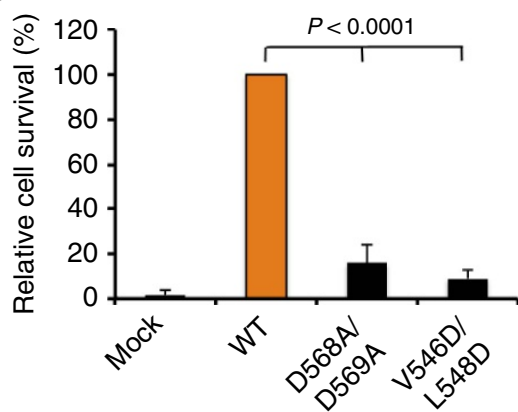

d
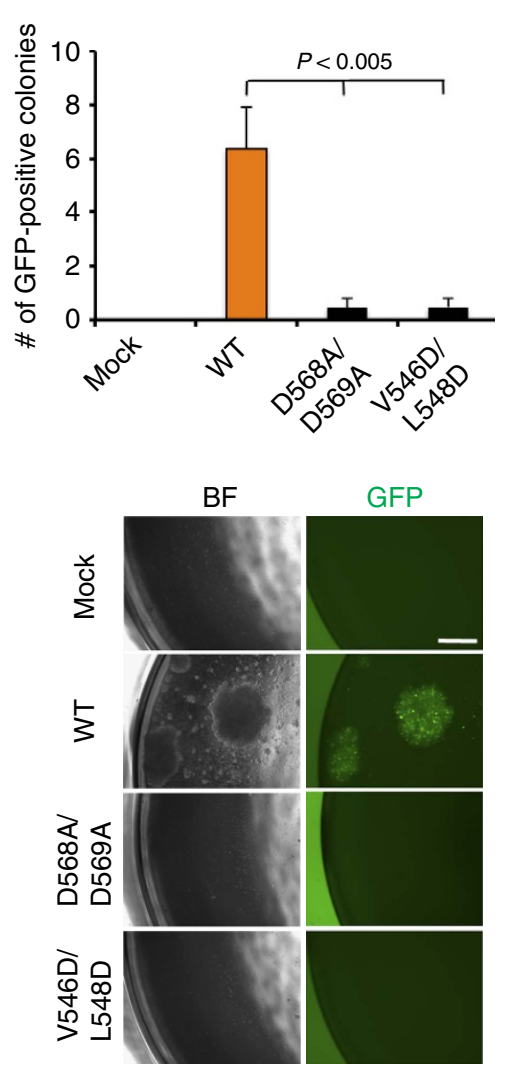

g

FLAG-BNRF1

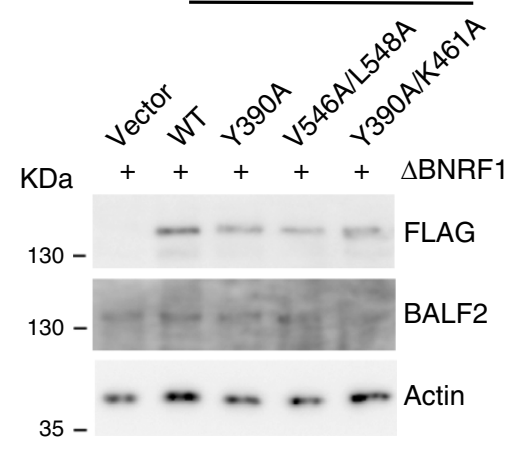


ImagePro Plus software (Media Cybernetics, Silver Spring, MD) and Adobe Photoshop CS5 for image processing. Transfected cells with four or more BNRF1 foci co-localizing with DAXX foci were scored as positive for BNRF1 nuclear co-localization with DAXX.

Subcellular fractionation assay. Hep2 cells were transfected in $6 \mathrm{~cm}$ plates with $2 \mu \mathrm{g}$ expression plasmids for either empty FLAG vector, or FLAG-tagged BNRF1 wt or mutants using Lipofectamine 2000 (Invitrogen). Cells were harvested $48 \mathrm{~h}$ post transfection and subject to REAP nuclear/cytoplasmic fractionation ${ }^{29}$. Briefly, cells were washed twice with ice-cold PBS, and collected with PBS into $1.5 \mathrm{ml}$ microcentrifuge tube. Cell pellets were triturated five times with $1 \mathrm{ml}$ ice-cold $0.1 \%$ NP40-PBS using p1000 micropipette. An aliquot $(200 \mu \mathrm{l})$ was removed into a fresh tube as the whole-cell extracts, and the rest of samples was centrifuged at max speed for $1 \mathrm{~min}$ at $4{ }^{\circ} \mathrm{C}$ with table top centrifuge. An aliquot $(200 \mu \mathrm{l})$ of supernatants was removed into a fresh tube as the cytoplasmic fraction, and the remainder of supernatants was removed by aspiration. The pellet was resuspended in $1 \mathrm{ml}$ ice-cold $0.1 \%$ NP40-PBS, and centrifuged at max speed for $1 \mathrm{~min}$ at $4{ }^{\circ} \mathrm{C}$ with table top centrifuge. The supernatant was removed, and the nuclear pellet was resuspended with $1 \mathrm{x}$ Laemmli buffer $(200 \mu \mathrm{l})$ and solubilized by brief sonication. The resulting cytoplasmic and nuclear fractions, along with the whole-cell extracts were analysed by WB.

EBV bacmids and virus production cells. Viruses were produced using chloramphenicol and hygromycin resistant bacmids containing the EBV genome and the gene coding for green fluorescence protein (GFP). 293/EBV-wt and $\triangle B N R F 1$ cells (gifts from H.J. Delecluse) are 293T cells transfected with the wild-type EBV bacmid or an EBV bacmid with the BNRF1 gene deleted ${ }^{17,30}$. EBV bacmids with D568A/D569A and V546D/L548D mutations in BNRF1 were generated from recombinant B95-8 and M81 genome, respectively by recombineering using two-step markerless red recombination method ${ }^{31}$. The mutations were confirmed by sequencing and restriction enzyme analysis with $E c o$ RI on $0.8 \%$ agarose gel. To further assure that the recombineering process did not alter the rest of the genome, the gel was transferred and assayed by Southern blot for the integrity of $\mathrm{Wp}$ and TR regions using ${ }^{32} \mathrm{P}$-labelled $\mathrm{Wp}$ - or TR-specific probes. The images were analysed by Phosphor-imager, visualized by Typhoon 9410 Imager (GE Healthcare), and analysed with ImageQuant 5.2 software (Molecular Dynamics).

BNRF1 wt and mutant production cells were generated by transfecting the bacmid DNA into 293T cells with Effectene transfection reagent (Qiagen) and selection with $100 \mu \mathrm{g} \mathrm{ml}^{-1}$ hygromycin for 3 weeks. 293/EBV-wt and 293/BNRF1 mutant cells were continuously grown in DMEM medium supplemented with $10 \%$ FBS and $100 \mu \mathrm{g} \mathrm{ml}^{-1}$ hygromycin. The populations of virus production cells were confirmed by visualization of GFP and EBNA1 protein by immunofluorescence as described above. Images were captured with a $60 \times$ objective lens on a Nikon E600 Uprigh microscope using ImagePro Plus software and Adobe Photoshop CS5 for image processing

Virus production and titre measurements. EBV lytic virus production was performed in 293/EBV wt and BNRF1 mutant cells co-transfected in $15 \mathrm{~cm}$ plates with expression plasmids for BALF4 $(3.5 \mu \mathrm{g})$ and HA-tagged BZLF1 $(7 \mu \mathrm{g})$ using $30 \mu \mathrm{l}$ of Lipofectamine 2000 (Invitrogen). For the production of BNRF1 complemented viruses, 293/ BNRF1 cells were co-transfected with expression plasmids for BALF4, BZLF1 and either empty FLAG vector or FLAG-tagged BNRF1 wt or mutants $(5 \mu \mathrm{g})$. At $24 \mathrm{~h}$ post transfection, a small fraction of transfected cells were replated into six-well plate and assayed by WB for viral reactivation at $72 \mathrm{~h}$ post transfection. The rest of transfected cells were plated into two $15 \mathrm{~cm}$ plates in $35 \mathrm{ml}$ culture medium and induced for 6 days before harvest for virus preparation. The media of virus production cells were harvested by centrifugation at about $2,000 \mathrm{~g}$ for $10 \mathrm{~min}$ and filtered through $0.45 \mathrm{~mm}$ filters. The concentrated virus were prepared by ultracentrifugation of the filtered media over $5 \mathrm{ml}$ layer of $10 \%$ sucrose in $1 \times$ PBS at $100,000 \mathrm{~g}$ for $1 \mathrm{~h}$ at $4{ }^{\circ} \mathrm{C}$ with a Sorvall WX
100 ultracentrifuge, resuspended in B cell growth medium with 1/100 volume of original medium, and stored at $-80^{\circ} \mathrm{C}$

Viral titres were assayed by real-time PCR detection of DNA copy number ${ }^{19}$. Viral DNA was extracted by treatment of virus-containing media with $10 \mathrm{U}$ per $50 \mu \mathrm{l}$ of DNase I (New England Biolabs) for $1 \mathrm{~h}$ at $37^{\circ} \mathrm{C}$, followed by $10 \mathrm{~min}$ heat inactivation at $70^{\circ} \mathrm{C}$ in the presence of $5 \mathrm{mM}$ EDTA. Samples were further treated with $0.1 \mathrm{mg} \mathrm{ml}^{-1}$ of proteinase $\mathrm{K}$ at $50^{\circ} \mathrm{C}$ for $1 \mathrm{~h}$, followed by $20 \mathrm{~min}$ of heat inactivation at $75^{\circ} \mathrm{C}$. The released viral DNA was measured by real-time PCR analysis, using a serial dilution series of Namalwa cell lysate as the standard curve, which contain two copies of integrated EBV genome per Namalwa cell. EBV genomes were detected using primers specific to EBER region (forward, 5'-GGACAGCCGTTGCCCTAGTGGTTT- $3^{\prime}$; reverse, $5^{\prime}$-CGATAAGCTTAAAAA TAGCGGACAAGCCG- $3^{\prime}$ ). Infection into Raji cells and WB of the concentrated virus were further used to examine the expression and packaging of BNRF1 proteins in corresponding virions. For Raji cells infection, mock medium or medium containing the concentrated virus was added into wells with about $2 \times 10^{5}$ Raji cells at a concentration of 30 viral DNA copies per cell in triplicates. A total of $100 \mathrm{ng} \mathrm{ml}^{-1}$ tetradecanoyl phorbol acetate was also added to each well to amplify the GFP signal. The viral infection was determined 4 days after infection by measuring the percentage of GFP-positive Raji cells by fluorescence-activated cell sorting analysis with an EPICS XL flow cytometer (Beckman Coulter). For WB of virions, the concentrated virus was lysed in $2 \times$ laemmli buffer, and protein gel loading volumes were normalized according to viral titres to ensure equal amounts of virion protein in each well. Equal volume of mock $293 \mathrm{~T}$ media was treated in the same procedure as viral production and the resulting concentrated sample was used as a negative control in the WB analysis.

Western blotting and antibodies. Equal amounts of samples in $1 \times$ Laemml buffer were electrophoresed on an 8-16\% Tris-Glycine gel (Life Technologies), transferred to nitrocellulose membrane, blotted with the indicated antibodies, and visualized by ECL plus kit (GE Healthcare) using LAS 3,000 imager (Fuji Film). The antibodies used in IF and WB were as followings: Rabbit polyclonal rabbit anti-DAXX (F7810, 1:1,000 dilution), mouse monoclonal anti-beta-Actin-Peroxidase (A33854, 1:2,000 dilution), mouse monoclonal anti-FLAG M2-Peroxidase (A8592, 1:2,000 dilution), and anti-FLAG M2 Affinity Gel (A2220) were purchased from Sigma-Aldrich. Mouse monoclonal anti-EAD (MAB8186, 1:500 dilution), anti-EBV VCA (MAB8184, 1:500 dilution), and rabbit polyclonal anti-Histone H3 (06-755, 1:1,000 dilution) were purchased from EMD Millipore. Rabbit polyclonal anti-GAPDH (ab9485, 1:1,000 dilution) was purchased from Abcam. Mouse monoclonal anti-EBNA1 (MCA2707, 1:600 dilution) was purchased from BioRad. Mouse monoclonal anti-HA (12CA5, 1:1,000 dilution) was from Roche. Rabbit anti-BNRF1 (1:100 dilution) and anti-BALF2 (1:200 dilution) were raised against the peptides from BNRF1 and BALF2, and custom produced by YenZym Antibodies, LLC.

Viral infection assay. Primary B cells were infected with concentrated EBV recombinant viruses at a ratio of 30 viral DNA copies per cell (multiplicity of infection of 30). Infected cells were harvested at specified time points for downstream assays. For measuring virus gene expression in infected B cells, cells were collected at $72 \mathrm{~h}$, or 1 week post infection, and total RNA was purified using Trizol reagent (Invitrogen) as manufacturer's instruction. The RNA samples were treated with DNase I for $45 \mathrm{~min}$ at $37^{\circ} \mathrm{C}$, followed by DNase I inactivation in the presence of EDTA at $65^{\circ} \mathrm{C}$ for $5 \mathrm{~min}$. cDNA was synthesized using the Super Script IV Reverse Transcriptase (Invitrogen). The resulting CDNA was then subject to real time PCR analysis with ABI Prism 7900 Sequence Detection System (Applied Biosystems). Relative PCR with reverse transcription was determined from at least three independent viral infections using $\triangle \mathrm{CT}$ methods relative to internal control GUSB gene. Real-time PCR primers used are as followings: EBNA1 (forward, $5^{\prime}$-GGTCGTGGACGTGGAGAAAA-3'; reverse, 5' -GGTGGAGACCCGGAT GATG-3'), EBNA2 (forward, 5'-GCTTAGCCAGTAACCCAGCACT-3'; reverse, $5^{\prime}$-TGCTTAGAAGGTTGTTGGCATG-3'), EBNA3C (forward, 5'-GCCGGGCT GTCAAGCA-3'; reverse, 5' -CCCACTATCGAGTATCAGGTTTGAT-3'), ZTA

Figure 6 | BNRF1-DAXX interaction is essential for EBV latent cycle gene expression during primary infection and B-cell immortalization. (a) Primary human B-lymphocytes were mock treated or infected with recombinant EBV wt (orange) or BNRF1 D568A/D569A genomes at a MOI of 30 for 1 week and then assayed by RT-PCR for EBV latency-associated genes EBNA1, EBNA2, EBNA3C or lytic immediate early gene ZTA, or cellular proliferation marker Ki67. The bar graph represents means \pm s.d. from at least three independent infection experiments. $P$ value was calculated by two-tailed $t$-test. (b) The same as in a, except that BNRF1 V546D/L548D mutant virus was used to infect primary B cells. (c) Resazurin assay measuring the relative cell survival for B-cells infected with mock, EBV wt, D568A/D569A or V546D/L548D recombinant virus at 4 weeks post infection. Data represent means $\pm s . d$ ( $n=3$ ), two-tailed $t$-test. (d) Colony formation assay for primary B cells infected with mock, EBV wt, D568A/D569A or V546D/L548D recombinant virus at 3 weeks post infection. Data represent means $\pm \mathrm{s} . \mathrm{d}(n=3)$, two-tailed $t$-test. (e) Representative images of the B-cell clonal proliferation used for quantification, as shown in d. BF indicates bright-field imaging, and scale bar, $200 \mu \mathrm{m}$. (f) Primary B-lymphocytes were infected at a MOI of 10 with recombinant EBV virus generated by trans-complementation of the BNRF1 deletion mutant ( $\triangle B N R F 1$ ) with either empty vector, FLAG-BNRF1 wt, Y390A, V546A/L548A or Y390A/K461A. EBNA1 and EBNA2 expression was assayed by quantitative real-time PCR at 1 week post infection. Data represent means \pm s.d from two independent experiments with triplicates. No RT controls shown in green. (g) WB for virions generated by trans-complementation with FLAG-BNRF1 wt or mutants, as indicated, and probed with FLAG, BLAF2 or actin. MOI, multiplicity of infection; RT-PCR, PCR with reverse transcription. 
(forward, 5' -TCTGAACTAGAAATAAAGCGATACAAGAA-3'; reverse, 5' -TTGG GCACATCTGCTTCAAC-3'), Ki67 (forward, 5'-ATGCAGACCCAGTGG ACACC-3'; reverse, 5' -TGCTGCCGGTTAAGTTCTCT- $3^{\prime}$ ), GUSB (forward, 5'-CG CCCTGCCTATCTGTATTC- $3^{\prime}$; reverse, $5^{\prime}$-TCCCCACAGGGAGTGTGTAG- ${ }^{\prime}$ ).

For measuring proliferation of infected cells, cells were assayed at 4 weeks post infection by resazurin viability assay. Briefly, infected cells were plated on 96-well plates in triplicates with at a density of 5,000 cells per well. The blank medium was used as negative control. After culture for $48 \mathrm{~h}$, resazurin was added into each well at a final concentration of $50 \mu \mathrm{M}$ to measure cell proliferation. The reads were performed at 6 and $24 \mathrm{~h}$ after adding resazurin by the use of Envision 2,104 Multilabel Reader (Perkin Elmer). The relative cell survival was calculated as percentage relative to the reads from wt EBV infection from at least three independent infection experiments.

For colony formation assay, about $1 \times 10^{3}$ infected cells were plated on 96-well plates at 1 week post infection and cultured for another 2 weeks. Colony images were acquired and processed using a Nikon TE2000-U Inverted microscope with $4 \times$ objective, Nikon DS-Ril digital camera and NIS-Elements D software. Statistical analysis was performed by two-tailed Student's $t$-test.

Data availability. Atomic coordinates and structure factors of the BNRF1 DID-DAXX HBD-H3.3-H4 complex have been deposited in the Protein Data Bank (PDB) under primary accession code 5KDM. The authors declare that all other data supporting the findings of this study are available within the article and its Supplementary Information file.

\section{References}

1. Alabert, C. \& Groth, A. Chromatin replication and epigenome maintenance. Nat. Rev. Mol. Cell Biol. 13, 153-167 (2012).

2. Avvakumov, N., Nourani, A. \& Cote, J. Histone chaperones: modulators of chromatin marks. Mol. Cell 41, 502-514 (2011).

3. Burgess, R. J. \& Zhang, Z. Histone chaperones in nucleosome assembly and human disease. Nat. Struct. Mol. Biol. 20, 14-22 (2013).

4. Gurard-Levin, Z. A., Quivy, J. P. \& Almouzni, G. Histone chaperones: assisting histone traffic and nucleosome dynamics. Annu. Rev. Biochem. 83, 487-517 (2014).

5. Ransom, M., Dennehey, B. K. \& Tyler, J. K. Chaperoning histones during DNA replication and repair. Cell 140, 183-195 (2010).

6. Filipescu, D., Muller, S. \& Almouzni, G. Histone H3 variants and their chaperones during development and disease: contributing to epigenetic control. Annu. Rev. Cell. Dev. Biol. 30, 615-646 (2014).

7. Maze, I., Noh, K. M., Soshnev, A. A. \& Allis, C. D. Every amino acid matters: essential contributions of histone variants to mammalian development and disease. Nat. Rev. Genet. 15, 259-271 (2014).

8. Drane, P., Ouararhni, K., Depaux, A., Shuaib, M. \& Hamiche, A. The death-associated protein DAXX is a novel histone chaperone involved in the replication-independent deposition of H3.3. Genes Dev. 24, 1253-1265 (2010).

9. Elsasser, S. J., Noh, K. M., Diaz, N., Allis, C. D. \& Banaszynski, L. A. Histone $\mathrm{H} 3.3$ is required for endogenous retroviral element silencing in embryonic stem cells. Nature 522, 240-244 (2015).

10. Goldberg, A. D. et al. Distinct factors control histone variant H3.3 localization at specific genomic regions. Cell 140, 678-691 (2010).

11. Lewis, P. W., Elsaesser, S. J., Noh, K. M., Stadler, S. C. \& Allis, C. D. Daxx is an H3.3-specific histone chaperone and cooperates with ATRX in replicationindependent chromatin assembly at telomeres. Proc. Natl Acad. Sci. USA 107, 14075-14080 (2010).

12. Wong, L. H. et al. ATRX interacts with $\mathrm{H} 3.3$ in maintaining telomere structural integrity in pluripotent embryonic stem cells. Genome Res. 20, 351-360 (2010).

13. Everett, R. D. \& Chelbi-Alix, M. K. PML and PML nuclear bodies: implications in antiviral defence. Biochimie 89, 819-830 (2007).

14. Lieberman, P. M. Chromatin structure of Epstein-Barr virus latent episomes. Curr. Top. Microbiol. Immunol. 390, 71-102 (2015).

15. Schreiner, S. \& Wodrich, H. Virion factors that target Daxx to overcome intrinsic immunity. J. Virol. 87, 10412-10422 (2013).

16. Knipe, D. M. et al. Snapshots: chromatin control of viral infection. Virology 435, 141-156 (2013).

17. Feederle, R. et al. Epstein-Barr virus BNRF1 protein allows efficient transfer from the endosomal compartment to the nucleus of primary B lymphocytes. J. Virol. 80, 9435-9443 (2006).

18. Tsai, K. et al. Viral reprogramming of the Daxx histone $\mathrm{H} 3.3$ chaperone during early Epstein-Barr virus infection. J. Virol. 88, 14350-14363 (2014).

19. Tsai, K., Thikmyanova, N., Wojcechowskyj, J. A., Delecluse, H. J. \& Lieberman, P. M. EBV tegument protein BNRF1 disrupts DAXX-ATRX to activate viral early gene transcription. PLoS Pathog. 7, el002376 (2011).

20. Tsai, K., Messick, T. E. \& Lieberman, P. M. Disruption of host antiviral resistances by gammaherpesvirus tegument proteins with homology to the FGARAT purine biosynthesis enzyme. Curr. Opin. Virol. 14, 30-40 (2015).
21. Elsässer, S. J. et al. DAXX envelops a histone H3. 3-H4 dimer for H3. 3-specific recognition. Nature 491, 560-565 (2012).

22. Liu, C. P. et al. Structure of the variant histone H3.3-H4 heterodimer in complex with its chaperone DAXX. Nat. Struct. Mol. Biol. 19, 1287-1292 (2012).

23. Anand, R., Hoskins, A. A., Stubbe, J. \& Ealick, S. E. Domain organization of Salmonella typhimurium formylglycinamide ribonucleotide amidotransferase revealed by X-ray crystallography. Biochemistry 43, 10328-10342 (2004)

24. Johannsen, E. et al. Proteins of purified Epstein-Barr virus. Proc. Natl Acad. Sci. USA 101, 16286-16291 (2004).

25. McCoy, A. J. et al. Phaser crystallographic software. J. Appl. Crystallogr. 40, 658-674 (2007).

26. Emsley, P. \& Cowtan, K. Coot: model-building tools for molecular graphics. Acta Crystallogr. D Biol. Crystallogr. 60, 2126-2132 (2004).

27. Adams, P. D. et al. PHENIX: building new software for automated crystallographic structure determination. Acta Crystallogr. D Biol. Crystallogr. 58, 1948-1954 (2002).

28. Lin, D. Y. \& Shih, H. M. Essential role of the $58-\mathrm{kDa}$ microspherule protein in the modulation of Daxx-dependent transcriptional repression as revealed by nucleolar sequestration. J. Biol. Chem. 277, 25446-25456 (2002).

29. Suzuki, K., Bose, P., Leong-Quong, R. Y., Fujita, D. J. \& Riabowol, K. REAP: a two minute cell fractionation method. BMC Res. Notes 3, 294 (2010).

30. Delecluse, H. J., Hilsendegen, T., Pich, D., Zeidler, R. \& Hammerschmidt, W. Propagation and recovery of intact, infectious Epstein-Barr virus from prokaryotic to human cells. Proc. Natl Acad. Sci. USA 95, 8245-8250 (1998).

31. Tischer, B. K., Smith, G. A. \& Osterrieder, N. En passant mutagenesis: a two step markerless red recombination system. Methods Mol. Biol. 634, 421-430 (2010).

\section{Acknowledgements}

We thank Henri-Jacques Delecluse for generously providing EBV wt and BNRF1 deletion mutant bacmids, and the Wistar Institute Imaging and Flow Cytometry Core Facility. We also thank Kevin Tsai and Lilian Chan for reagents, and helpful discussions. This work was supported by NIH RO1 (DE 017336, CA 093606, P01 CA174439) to P.M.L. and by the Wistar Cancer Center core grant (P30 CA10815), as well as by the Leukemia and Lymphoma Society and STARR Foundation grants to D.J.P. and by the Memorial Sloan-Kettering Cancer Center Core Grant (P30 CA008748). The crystallographic research was conducted at the Northeastern Collaborative Access Team beamlines, which are funded by NIGMS (P41 GM103403). The Pilatus 6M detector on 24-ID-C beam line is funded by a NIH-ORIP HEI grant (S10 RR029205). This research used resources of the Advanced Photon Source, the US Department of Energy (DOE) Office of Science User Facility operated for the DOE Office of Science by Argonne National Laboratory under Contract No. DE-AC02-06CH11357.

\section{Author contributions}

H.H. generated the BNRF1 DID-DAXX HBD-H3.3-H4 complex, solved the structure and undertook the in vitro mutational studies under the supervision of D.J.P. A.W. and F.L. generated BNRF1 mutant bacmids and 293T virus production cells. O.V. performed IF experiments. Z.D. carried Co-IP, viral production and infection, and other functional studies. H.H., Z.D., P.M.L. and D.J.P. analysed the data, prepared the figures and wrote the manuscript.

\section{Additional information}

Supplementary Information accompanies this paper at http://www.nature.com/ naturecommunications

Competing financial interests: The authors declare no competing financial interests.

Reprints and permission information is available online at http://npg.nature.com/ reprintsandpermissions/

How to cite this article: Huang, H. et al. Structural basis underlying viral hijacking of a histone chaperone complex. Nat. Commun. 7:12707 doi: 10.1038/ncomms12707 (2016).

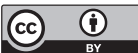

This work is licensed under a Creative Commons Attribution 4.0 International License. The images or other third party material in this article are included in the article's Creative Commons license, unless indicated otherwise in the credit line; if the material is not included under the Creative Commons license, users will need to obtain permission from the license holder to reproduce the material. To view a copy of this license, visit http://creativecommons.org/licenses/by/4.0/

(C) The Author(s) 2016 